Pacific Journal of Mathematics

MEASURES WHOSE RANGE IS A BALL 


\section{MEASURES WHOSE RANGE IS A BALL}

NEIL W. RICKERT

It has been shown by R. P. Kaufman and the author that if $\mu$ is a measure of total variation 1 with values in $R^{n}$, then there is a measurable set $E$ with

$$
|\mu(E)| \geqq \frac{1}{2 \pi^{1 / 2}} \frac{\Gamma(n / 2)}{\Gamma((n+1) / 2)} .
$$

The main purpose of this paper is to determine for which measures $\mu$ there is no set $E$ with

$$
|\mu(E)|>\frac{1}{2 \pi^{1 / 2}} \frac{\Gamma(n / 2)}{\Gamma((n+1) / 2)} .
$$

It will be shown that they are the measures which satisfy the following two conditions:

(i) The measure of the whole space is zero.

(ii) The induced probability measure $\alpha \circ f(|\mu|)$ on the projective space $P^{n-1}$ is orthogonally invariant, where $f=$ $d \mu / d|\mu|$ maps the measure space to the sphere $S^{n-1}$ and $\alpha$ is the natural map of $S^{n-1}$ onto $P^{n-1}$.

A different, more geometric proof of the first inequality above has been given by Schwarz [5].

It is clear that condition (i) is equivalent to the centre of the range of $\mu$ being 0 . It will be shown that for $n \geqq 2$, condition (ii) is equivalent to the range of $\mu$ being a ball of radius

$$
\frac{1}{2 \pi^{1 / 2}} \frac{\Gamma(n / 2)}{\Gamma((n+1) / 2)} .
$$

Of course for $n=1$ condition (ii) is trivially satisfied by every measure with range in $R^{1}$ since $P^{0}$ consists of one point.

Let $X$ be a space, $\Sigma$ a $\sigma$-field of subsets of $X$, and $\mu$ a measure on $\Sigma$ with values in $R^{n}$. By the range of $\mu$ we mean the set $\{\mu(E) ; E \in \Sigma\}$. For $E$ in $\Sigma,|\mu|(E)$ will denote the total variation of $\mu$ on the set $E$. Note that $|\mu|$ is a positive measure on $\Sigma \cdot\|\mu\|$ will denote $|\mu|(x)$.

If $X^{\prime}$ is another space, and $\Sigma^{\prime}$ a $\sigma$-field of subsets of $X^{\prime}$, and if $f$ is a map of $X$ into $X^{\prime}, f$ will be called measurable if $f^{-1}(E) \in \Sigma$ whenever $E \in \Sigma^{\prime}$. If $f$ is measurable, and if $\mu$ is a measure on $\Sigma$, then $f(\mu)$ is that measure on $\Sigma^{\prime}$ defined by $f(\mu)(E)=\mu\left(f^{-1}(E)\right)$. When $X^{\prime}$ is a topological space (such as a sphere or a projective space) we shall always understand that we are using the $\sigma$-field of Borel sets. By abuse of language we shall speak of measures on $X$, or 
measures on $X^{\prime}$ when we should be speaking of measures on $\Sigma$ on $\Sigma^{\prime}$.

If $f$ denotes the Radon-Nikodym derivative $d \mu / d|\mu|, f$ is a measurable map of $X$ into $R^{n}$. By redefining $f$ on a set of $|\mu|$ measure zero if necessary, we may assume that $f$ maps $X$ into the sphere $S^{n-1}$. Thus $f(\mu)$ and $f(|\mu|)$ are Borel measures on $S^{n-1}$. Note that $f(\mu)$ and $f(|\mu|)$ are related by $f(\mu)(d x)=x f(|\mu|)(d x)$. It is easily seen from this that $|f(\mu)|=f(|\mu|)$ and $\|f(\mu)\|=\|\mu\|$.

If $x \in S^{n-1}$ we denote by $H_{x}$ the hemisphere determined by $x$. That is $H_{x}=\left\{y \in S^{n-1} ;\langle y, x\rangle \geqq 0\right\}$. For $\mu$ an $R^{n}$ valued measure on $X$ we denote by $\rho_{\mu}$ the function defined on $S^{n-1}$ by $\rho_{\mu}(x)=\left\langle\mu\left(f^{-1}\left(H_{x}\right)\right), x\right\rangle$ where $f=d \mu / d|\mu|$.

Fix an arbitrary point $x_{0}$ in $S^{n-1}$. We denote by $G$ the group $S O(n)$ and $K$ the subgroup of $G$ consisting of those elements which fix $x_{0}$. If we choose an orthonormal basis $e_{1}, e_{2}, \cdots, e_{n}$ for $R^{n}$ such that $e_{1}=x_{0}$, then $G$ consists of orthogonal metrices of determinant 1 , while $K$ consists of matrices

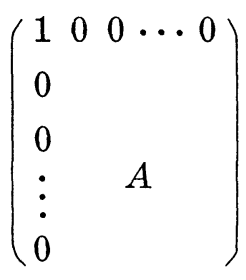

where $A$ is an $(n-1) \times(n-1)$ orthogonal matrix of determinant 1 . The projective space $P^{n-1}$ is the space obtained from $S^{n-1}$ by identifying antipodal points. We shall denote by $\alpha$ the natural projection of $S^{n-1}$ onto $P^{n-1}$.

We denote by $m$ the unique probability measure on $S^{n-1}$ which is invariant under orthogonal transformations. Up to a scalar factor $m$ is the usual surface measure on $S^{n-1}$. Note that $\alpha(m)$ is the invariant probability measure on $P^{n-1}$.

Lemma 1. $\rho_{\mu}$ is a continuous function on $S^{n-1}$ and

$$
\int_{S^{n-1}} \rho_{\mu}(x) m(d x)=\frac{1}{2 \pi^{1 / 2}} \frac{\Gamma(n / 2)}{\Gamma((n+1) / 2)}
$$

if $\mu$ is an valued measure of total variation 1 on $X$.

Proof. The second assertion was essentially proved in the proof of Theorem 3 of [3]. For the first assertion observe that

$$
\begin{aligned}
& \rho_{\mu}(x)-\rho_{\mu}(y)=\left\langle x, \mu\left(f^{-1}\left(H_{x}\right)\right)\right\rangle-\left\langle y, \mu\left(f^{-1}\left(H_{y}\right)\right)\right\rangle \\
& \quad=\int_{X} \max (0,\langle x, f(t)\rangle)|\mu|(d t)-\int_{X} \max (0,\langle y, f(t)\rangle)|\mu|(d t) \\
& \quad \leqq \int_{X}\langle x-y, f(t)\rangle|\mu|(d t) \leqq|x-y| .
\end{aligned}
$$


The continuity of $\rho_{\mu}$ follows immediately.

LEMma 2. Let $\lambda$ be a probability measure on $S^{n-1}$ and suppose that $\left\langle x, \int_{H_{x}} y \lambda(d y)\right\rangle$ is independent of $x$. Suppose furthermore that $\lambda$ is K-invariant (i.e. $\lambda(k E)=\lambda(E)$ for $k \in K)$. Then $\alpha(\lambda)$ is the invariant probability measure on $P^{n-1}$.

The proof of Lemma 2 will require some properties of spherical functions, so we postpone the proof until the end of this paper.

Lemma 3. Let $\mu$ be an $R^{n}$ valued measure of total variation 1 on $\Sigma$, a $\sigma$-field of subsets of $X$. Then a necessary and sufficient condition that $\rho_{\mu}$ be a constant function on $S^{n-1}$ is that $\mu(X)=0$ and $\alpha\left(f(|\mu|)\right.$ is the invariant probability measure on $P^{n-1}$.

Proof. Suppose first that $\mu(X)=0$ and $\alpha(f(|\mu|)$ is the invariant probability measure on $P^{n-1}$. Let $x$ be an element of $S^{n-1}$. Define the function $h$ on $P^{n-1}$ by $h(\alpha(y))=\langle x, y\rangle$ if $y \in H_{x}$. Note that if $y$ and $z$ are in $H_{x}$, and if $\alpha(y)=\alpha(z)$ it follows that $\langle x, y\rangle=0=\langle x, z\rangle$ whence it follows that $h$ is well defined. Since $\alpha(f(|\mu|))=\alpha(m)$ it follows that

$$
\begin{aligned}
& \frac{1}{\pi^{1 / 2}} \frac{\Gamma(n / 2)}{\Gamma((n+1) / 2)}=\int_{P^{n-1}} h(p) \alpha(m)(d p) \\
& \quad=\int_{P^{n-1}} h(p) \alpha(f(|\mu|)(d p) \\
& \quad=\int_{H_{x}}\langle x, y\rangle f(|\mu|)(d y)-\int_{H_{-x}}\langle x, y\rangle f(|\mu|)(d y) \\
& \quad=\left\langle x, \int_{H_{x}} y f(|\mu|)(d y)\right\rangle+\left\langle-x, \int_{H_{-x}} y f(|\mu|)(d y)\right\rangle \\
& =\rho_{\mu}(x)+\rho_{\mu}(-x) .
\end{aligned}
$$

Also $\rho_{\mu}(x)-\rho_{\mu}(-x)=\langle\mu(X), x\rangle=0$. Thus

$$
\rho_{\mu}(x)=\frac{1}{2 \pi^{1 / 2}} \frac{\Gamma(n / 2)}{\Gamma((n+1) / 2)}
$$

and $\rho_{\mu}$ is a constant function.

Conversely suppose that $\rho_{\mu}$ is constant. For $x \in S^{n-1}$, since

$$
\begin{aligned}
& \left\langle x, \mu\left(f^{-1}\left(H_{x} \cap H_{-x}\right)\right\rangle=0,\right. \\
\langle x, \mu(X)\rangle & =\left\langle x, \mu\left(f^{-1}\left(H_{x}\right)\right)\right\rangle+\left\langle x, \mu\left(f^{-1}\left(H_{-x}\right)\right)\right\rangle \\
& =\rho_{\mu}(x)-\rho_{\mu}(-x)=0 .
\end{aligned}
$$

Thus $\mu(X)=0$. 
Now let $\lambda$ be the probability measure on $S^{n-1}$ defined by

$$
\lambda(E)=\int_{K} f(|\mu|)(k E) d k,
$$

so that if $\varphi$ is a continuous function on $S^{n-1}$,

$$
\int_{S^{n-1}} \varphi(y) \lambda(d y)=\int_{K} \int_{S^{n-1}} \varphi\left(k^{-1} y\right) f(|\mu|)(d y) d k .
$$

Note that $\lambda$ is $K$-invariant, and that

$$
\begin{aligned}
& \left\langle x, \int_{H_{x}} y \lambda(d y)\right\rangle=\int_{H_{x}}\langle x, y\rangle \lambda(d y)=\int_{S^{n-1}} \max (0,\langle x, y\rangle) \lambda(d y) \\
& =\int_{K} \int_{S^{n-1}} \max \left(0,\left\langle x, k^{-1} y\right\rangle\right) f(|\mu|)(d y) \\
& =\int_{K} \int_{S^{n-1}} \max (0,\langle k x, y\rangle) f(|\mu|)(d y)=\int_{K} \rho_{\mu}(k x) d k .
\end{aligned}
$$

Since $\rho_{\mu}$ is constant it follows that $\lambda$ satisfies the hypotheses of Lemma 2. We may thus conclude that $\alpha(\lambda)$ is the invariant measure $\alpha(m)$ on $P^{n-1}$. We then conclude that if $h$ is a continuous function on $P^{n-1}$ whose value at a point $y$ depends only on the distance from $y$ to $\alpha\left(x_{0}\right)$

$$
\int h(y) \alpha(f(|\mu|))(d y)=\int h(y) \alpha(\lambda) d y=\int h(y) \alpha(m) d y .
$$

By $x_{0}$ was an arbitrary point of $S^{n-1}$, so the same assertion is true as long as the value of $h$ at $y$ depends only on the distance from $y$ to some point $x$ in $P^{n-1}$ (where $x$ depends on $h$ but not $y$ ). But linear combinations of such continuous functions are dense in all continuous functions on $P^{n-1}$ (see [4]) so we conclude that $\alpha(f(|\mu|))=\alpha(m)$, as required.

THEOREM 1. Let $\mu$ be an $R^{n}$-valued measure $(n \geqq 2)$ of total variation 1 on $\Sigma$, a $\sigma$-field of subsets of $X$. Then the following conditions are equivalent:

(1) If $f=d \mu / d|\mu|$ and $\alpha$ is the natural projection of $S^{n-1}$ onto $P^{n-1}$, then $\mu(X)=0$ and $\alpha(f(|\mu|))$ is the invariant measure on $P^{n-1}$.

(2) The range of $\mu$ is the ball with centre 0 and radius

$$
\frac{1}{2 \pi^{1 / 2}} \frac{\Gamma(n / 2)}{\Gamma((n+1) / 2)} .
$$

(3) The convex hull of the range of $\mu$ is a ball with centre 0 .

(4) For each $E \in \Sigma,|\mu(E)| \leqq\left(1 / 2 \pi^{1 / 2}\right)(\Gamma(n / 2) / \Gamma((n+1) / 2)$. 
Proof. $\quad(4) \Rightarrow(1)$. It follows from (4) that

$$
\rho_{\mu}(x) \leqq \frac{1}{2 \pi^{1 / 2}} \frac{\Gamma(n / 2)}{\Gamma((n+1) / 2)}
$$

for $x \in S^{n-1}$. But $\rho_{\mu}$ is continuous, and

$$
\int_{S^{n-1}} \rho_{\mu}(x) m(d x)=\frac{1}{2 \pi^{1 / 2}} \frac{\Gamma(n / 2)}{\Gamma((n+1) / 2)}
$$

as was shown in Lemma 1. It follows that $\rho_{\mu}$ is constant, and (1) now follows from Lemma 3.

$(1) \Rightarrow(4)$. Assume that (1) is true. Suppose that there is a set $E \in \Sigma$ with $|\mu(E)|>\left(1 / 2 \pi^{1 / 2}\right)(\Gamma(n / 2) / \Gamma((n+1) / 2))$. Then there is an $x \in S^{n-1}$ such that $\langle x, \mu(E)\rangle>\left(1 / 2 \pi^{1 / 2}\right)(\Gamma(n / 2) / \Gamma((n+1) / 2))$. But clearly $\left\langle x, \mu\left(f^{-1}\left(H_{x}\right)\right)\right\rangle \geqq\langle x, \mu(E)\rangle$ so $\rho_{\mu}(x)>\left(1 / 2 \pi^{1 / 2}\right)(\Gamma(n / 2) / \Gamma((n+1) / 2))$ contradicting what was proved in Lemma 3 . It follows that (4) must be true.

$(1) \Rightarrow(2)$. From the implication $(1) \Rightarrow(4)$ we know that the range of $\mu$ is contained in the ball with centre 0 and radius

$$
\frac{1}{2 \pi^{1 / 2}} \frac{\Gamma(n / 2)}{\Gamma((n+1) / 2)} .
$$

Also from (1) it follows that $\mu$ is an atom free measure, since $\alpha(f(|\mu|))$ is atom free. Hence the range of $\mu$ is convex (see [1]). It suffices to show therefore that every point on the surface of the ball is in the range of $\mu$. But from (1) and Lemma 3 it follows that for $x \in S^{n-1}, \rho_{\mu}(x)=\left(1 / 2 \pi^{1 / 2}\right)(\Gamma(n / 2) / \Gamma((n+1) / 2))$. Whence we conclude, using (4), that

$$
\mu\left(f^{-1}\left(H_{x}\right)\right)=\frac{1}{2 \pi^{1 / 2}} \frac{\Gamma(n / 2)}{\Gamma((n+1) / 2)} x .
$$

Thus (2) is true.

$(2) \Longrightarrow(3)$. Obvious.

$(3) \Rightarrow(1)$. Assume that the convex hull of the range of $\mu$ is the ball with centre 0 , radius $r$. Since the range of $\mu$ is closed (see [1]) it includes every extreme point $r x$, for $x \in S^{n-1}$. But if $\mu(E)=r x$,

$$
\left\langle x, \mu\left(f^{-1}\left(H_{x}\right)\right)\right\rangle \geqq\langle x, \mu(E)\rangle=r .
$$

On the other hand $\left|\mu\left(f^{-1}\left(H_{x}\right)\right)\right| \leqq r$, so it follows that $\rho_{\mu}(x)=r$ for $x \in S^{n-1}$. Applying Lemma 3 , we see that (1) is true.

REMARK. From the implication $(3) \Longrightarrow(2)$ of the above theorem, it follows that if the convex hull of the range of a measure is a ball with centre 0 , then the measure is nonatomic, and the range is 
actually convex. In fact this can be seen directly, and does not depend on the centre of the ball being 0 . For if there were an atom, the convex hull of the range of $\mu$ would have a straight edge - that is there would be a line segment on the boundary of the convex hull of the range. This is not the case when the convex hull of the range is a ball, whence we conclude that $\mu$ is atom free, and has a convex range.

We now turn our attention to characterizing measures with range a ball whose centre need not be 0 .

Lemma 4. If $\mu$ is an $R^{n}$ valued measure on $X$, then the range of $\mu$ contains 0 and is symmetric about $\mu(X) / 2$.

Proof. The measure of the empty set is 0 , and the map

$$
\mu(E) \longrightarrow \mu(X-E)
$$

is a symmetry of the range of $\mu$ about $\mu(X) / 2$.

Lemma 5. Assume $\mu$ is an $R^{n}$ valued measure on $X$, and that $F$ is a measurable set. Define the measure $\lambda$ on $X$ by

$$
\lambda(E)=\mu(E-F)-\mu(E \cap F) .
$$

Set $f=d \mu / d|\mu|$ and $g=d \lambda / d|\lambda|$. Then $\alpha(f(|\mu|))=\alpha(g(|\lambda|))$.

Proof. Clearly $|\mu|=|\lambda|$ and $g(t)=f(t)$ or $g(t)=-f(t)$ depending on whether $t \in X-F$ or $t \in F$. Since $\alpha(x)=\alpha(-x)$ it follows that $\alpha(f(t))=\alpha(g(t))$ for $t \in X$. Since $|\mu|=|\lambda|$ the result follows.

THEOREM 2. Let $\mu$ be an $R^{n}$ valued measure on $X$, and define $f=d \mu / d|\mu|$. Let $\alpha$ be the natural projection of $S^{n-1}$ onto $P^{n-1}$. A necessary and sufficient condition that the range of $\mu$ be a ball is that the measure $\alpha(f(|\mu|))$ on $P^{n-1}$ be invariant under orthogonal transformations. In this case the centre of the ball is $\mu(X) / 2$ and its radius is $\left(1 / 2 \pi^{1 / 2}\right)(\Gamma(n / 2) / \Gamma((n+1) / 2))\|\mu\|$.

Proof. Without loss of generality we may assume that $\|\mu\|=1$. We may also assume that the range of $\mu$ is convex (see the remark following Theorem 1). Thus there is a measurable set $F$ with $\mu(F)=\mu(X) / 2$. Define $\lambda$ by

$$
\lambda(E)=\mu(E-F)-\mu(E \cap F) .
$$

Notice that if $E$ is any measurable set, 


$$
\lambda((E-F) \cup(F-E))=\mu(E)-\mu(X) / 2
$$

and

$$
\lambda(E)=\mu((E-F) \cup(F-E))-\mu(X) / 2 .
$$

It follows that the range of $\lambda$ is just the range of $\mu$ translated by $-\mu(X) / 2$. Note also that $\lambda(X)=0$. The result now follows easily from Theorem 1 and Lemmas 4 and 5 .

We turn our attention now to the proof of Lemma 2. We shall need to investigate certain properties of spherical functions. A more general discussion of spherical functions on spheres can be found in [4] and [2]. $\quad S^{n-1}$ is a symmetric space (seen [2] for the definition of symmetric spaces) and can be written as $G / K$ where $G$ and $K$ are the groups introduced earlier in the paper. Likewise $P^{n-1}$ is a symmetric space. For technical reasons part of our discussion will apply only to the case $n \geqq 3$, although the arguments could be suitably modified to apply to the case $n=2$. In any case Lemma 2 was already proved for the case $n=2$ in [3].

Since the $G$-invariant differential operators on $S^{n-1}$ are all polynomials in the Laplace-Beltrami operator $\Delta$ (see [2] p. 397), a function $f$ on $S^{n-1}$ is a spherical function if and only if

(i) $f$ is $K$-invariant (that is $f(k x)=f(x)$ for $k \in K$ ).

(ii) $f\left(x_{0}\right)=1$.

(iii) $f$ is an eigenfunction of the operator $\Delta$.

To determine the spherical functions we coordinatize $S^{n-1}$ as follows. A point $x$ in $S^{n-1}$ is given coordinates $\left(r, z_{1}, z_{2}, \cdots, z_{n-2}\right)$. Here $r$ is the distance from $x_{0}$ to $x$ measured along the surface of $S^{n-1}$ (that is $r$ is the angle between the vectors $x_{0}$ and $x$, so that $\left\langle x_{0}, x\right\rangle=\cos r$ ). If we project $x$ onto the $n-1$ dimensional plane orthogonal to the vector $x_{0}$, and then produce the corresponding vector until it intersects the sphere in the plane, we obtain a point in $S^{n-2}$. Then $\left(z_{1}, \cdots, z_{n-2}\right)$ are coordinates of this point in some local coordinates for $S^{n-2}$. In this way we obtain coordinates for $S^{n-1}$ except at $r=0$ or $r=\pi$.

Assume that on $S^{n-2}$ the Riemannian metric is $\sum a_{i j} d z_{i} d z_{j}$. Then clearly the Riemannian metric on $S^{n-1}$ is $d r^{2}+(\sin r)^{2} \sum a_{i j} d z_{i} d z_{j}$. Denoting by $\left(b_{i j}\right)$ the $(n-2) \times(n-2)$ matrix inverse to $\left(a_{i j}\right)$ the Laplace-Beltrami operator is given by

$$
\Delta f=\frac{1}{(\sin r)^{n-2}} \frac{\partial}{\partial r}\left((\sin r)^{n-2} \frac{\partial f}{\partial r}\right)+\frac{1}{(\sin r)^{2} A} \sum_{k} \frac{\partial}{\partial z_{k}} \sum_{j} b_{j k} A \frac{\partial f}{\partial z_{j}}
$$

where $A^{2}=\operatorname{det}\left(a_{i j}\right)$.

But if $f$ is $K$-invariant it is a function only of $r$ and we then have 


$$
\Delta f=\frac{\partial^{2} f}{\partial r^{2}}+(n-2) \frac{\cos r}{\sin r} \frac{\partial f}{\partial r} .
$$

Now if $f$ is a spherical function it satisfies $\Delta f+\lambda f=0$ for some complex number $\lambda$. Making a change of variables, we set $f(r)=$ $\varphi(\cos r)$ for some function $\varphi$. Then if $f$ is a spherical function $\varphi$ will satisfy

$$
(1-t)^{2} \frac{d^{2} \varphi}{d t^{2}}-(n-1) t \frac{d \varphi}{d t}+\lambda \varphi=0
$$

On the other hand suppose that $\varphi$ is twice continuously differentiable on $[-1,1]$ and satisfies the above differential equation. The function $f$ defined by $f(x)=\varphi(\cos r)=\varphi\left(\left\langle x_{0}, x\right\rangle\right)$ will then be twice continuously differentiable on $S^{n-1}$ and except at $x_{0}$ and $-x_{0}$ it will satisfy

$$
\Delta f+\lambda f=0 .
$$

By continuity this equation will also be satisfied at the exceptional points. From the proof on p. 400 of [2] it can be seen that $f\left(x_{0}\right) \neq 0$ unless $f$ vanishes identically, so after a suitable normalization $f$ is a spherical function. We therefore seek solutions of the equation

$$
\left(1-t^{2}\right) \frac{d^{2} \varphi}{d t^{2}}-(n-1) t \frac{d \varphi}{d t}+\lambda \varphi=0 \text {. }
$$

If $\phi$ is a polynomial of degree $k$ then necessarily $\lambda=k(n+k-2)$ as is readily verified by checking the term of degree $k$. If we can show that for every nonnegative integer $k$ there is a polynomial $\varphi_{k}^{n-1}$ of degree $k$ which satisfies the equation with $\lambda=k(n+k-2)$ and such that $\varphi_{k}^{n-1}(1)=1$ (this last condition is equivalent to the corresponding spherical function being 1 at $x_{0}$, and can be achieved by a suitable normalization), then these $\varphi_{k}^{n-1}$ will give rise to spherical functions on $S^{n-1}$. Furthermore using essentially the argument on p. 404 of [2] it can be deduced that all spherical functions arise in this way.

Notice that $\varphi_{k}^{1}$ satisfies $\varphi_{k}^{1}(\cos r)=\cos k r$. Thus

$$
\varphi_{k}^{1}(t)=\sum_{j \leqq k / 2}\left(2_{j}^{k}\right) t^{k-2 j}\left(t^{2}-1\right)^{j} .
$$

Notice also that $\varphi_{k}^{2}$ is just the Legendre polynomial

$$
\varphi_{k}^{2}(t)=(\text { constant })\left(\frac{d}{d t}\right)^{k}\left(t^{2}-1\right)^{k} .
$$

By differentiating the differential equation, observe that

$$
\varphi_{k-1}^{n+2}(t)=(\text { constant }) \frac{d}{d t} \varphi_{k}^{n}(t) .
$$


Thus we have proved

THEOREM 3. The spherical functions on $S^{n-1}$ are the functions $f_{k}^{n-1}(x)=\varphi_{k}^{n-1}\left(\left\langle x_{0}, x\right\rangle\right)$ where $\varphi_{k}^{n-1}$ satisfies

$$
\left(1-t^{2}\right) \frac{d^{2} \varphi}{d t^{2}}-(n-1) t \frac{d \varphi}{d t}+k(n+k-2) \varphi=0 .
$$

$\varphi_{k}^{n-1}$ is a polynomial of degree $k$ and $\varphi_{k}^{n-1}(1)=1$. Furthermore $\varphi_{k}^{n-1}$ is an odd polynomial if $k$ is odd, and is an even polynomial if $k$ is even. If $k$ is even (respectively odd) and if $j$ is an even (respectively odd) integer, $0 \leqq j \leqq k$, then the coefficient of $t^{j}$ in the polynomial $\varphi_{k}^{n-1}(t)$ does not vanish. In particular $\varphi_{k}^{n-1}(0) \neq 0$ if $k$ is even.

If $\mu$ is a $K$-invariant measure on $S^{n-1}$ we recall that its FourierStieltjes coefficients are defined by $\hat{\mu}\left(f_{k}^{n-1}\right)=\int f_{l}^{n-1}(x) \mu(d x)$ (see [2]). Likewise the Fourier coefficients of $K$-invariant functions on $S^{n-1}$ can be defined. We wish to investigate the Fourier coefficients of the function $\psi$ defined by $\psi(x)=\max \left(0,\left\langle x_{0}, x\right\rangle\right)$.

LeMma 6. For $n \geqq 3, \hat{\psi}\left(f_{k}^{n-1}\right) \neq 0$ if $k$ is even.

Proof. Writing $f$ for $f_{k}^{n-1}$ and $\varphi$ for $\varphi_{k}^{n-1}$ we have

$$
\begin{aligned}
\hat{\psi}(f) & =\int_{S^{n-1}} \psi(x) f(x) m(d x) \\
& =\int_{S^{n-1}} \max (0, \cos r) \varphi(\cos r) m(d x) \\
& =K \int_{0}^{\pi} \max (0, \cos r) \varphi(\cos r)(\sin r)^{n-2} d r
\end{aligned}
$$

where $K$ is chosen so that $K \int_{0}^{\pi}(\sin r)^{n-2} d r=1$. Thus

$$
\begin{aligned}
\hat{\psi}(f) & =K \int_{-1}^{1} \max (0, t) \varphi(t)\left(1-t^{2}\right)^{(n-3) / 2} d t \\
& =K \int_{0}^{1} t \varphi(t)\left(1-t^{2}\right)^{(n-3) / 2} d t .
\end{aligned}
$$

Integrating twice by parts yields

$$
\begin{aligned}
& \int_{0}^{1} t \varphi(t)\left(1-t^{2}\right)^{(n-3) / 2} d t \\
& =\frac{1}{n-1} \varphi(0)+\frac{1}{n-1} \int_{0}^{1}\left(1-t^{2}\right)^{(n-1) / 2} \varphi^{\prime}(t) d t \\
& =\frac{1}{n-1} \varphi(0)+\frac{1}{n-1} \int_{0}^{1} t\left(1-t^{2}\right)^{(n-1) / 2} \varphi^{\prime \prime}(t) d t+\int_{0}^{1} t^{2}\left(1-t^{2}\right)^{(n-3) / 2} \varphi^{\prime}(t) d t .
\end{aligned}
$$


Substituting from the differential equation

$$
\left(1-t^{2}\right) \varphi^{\prime \prime}(t)-(n-1) t \varphi^{\prime}(t)+k(n+k-2) \varphi(t)=0
$$

we see that

$$
(1-k(n+k-2) /(n-1)) \int_{0}^{1} t \varphi(t)\left(1-t^{2}\right)^{(n-3) / 2} d t=\frac{1}{n-1} \varphi(0) .
$$

But when $k$ is even, $\varphi(0)=\varphi_{k}^{n-1}(0) \neq 0$ so the desired conclusion follows.

We now proceed with the proof of Lemma 2. Thus assume that $\lambda$ is a $K$-invariant probability measure on $S^{n-1}$ such that

$$
\left\langle x, \int_{H_{x}} y \lambda(d y)\right\rangle
$$

is independent of $x$. Let $K^{\prime}$ be the subgroup of $G$ consisting of elements of $G$ which map $x_{0}$ into $-x_{0} . P^{n-1}$ is then the symmetric space $G / K^{\prime}$. Since $\lambda$ is $K$-invariant it is clear that $\alpha(\lambda)$ is $K^{\prime}$-invariant. Thus to show that $\alpha(\lambda)$ and $\alpha(m)$ are equal it suffices to show that they have the same Fourier-Stieltjes coefficients. But if $h$ is a spherical function on $P^{n-1}$ it is clear that the map $x \rightarrow h(\alpha(x))$ defines a spherical function on $S^{n-1}$. It follows then that the spherical functions on $P^{n-1}$ are given by $h(\alpha(x))=f_{k}^{n-1}(x)$ for $k$ even. To prove that $\alpha(\lambda)=\alpha(m)$ it thus suffices to show that $\hat{\lambda}\left(f_{k}^{n-1}\right)=\hat{m}\left(f_{k}^{n-1}\right)$ for $k$ even. But $\hat{m}\left(f_{0}^{n-1}\right)=\hat{\lambda}\left(f_{0}^{n-1}\right)=1$ since both are probability measures, and $\hat{m}\left(f_{k}^{n-1}\right)=0$ for $k$ a positive integer. We must therefore show that $\hat{\lambda}\left(f_{k}^{n-1}\right)=0$ for $k$ an even positive integer. Denote by $\mu$ the right $K$-invariant measure on $G$ which projects to the measure $\lambda$ on $S^{n-1}$. (In the notation of [3] $\mu=\tilde{\lambda}$ ). Then

$$
\begin{aligned}
\langle x, & \left.\int_{H_{x}} y \lambda(d y)\right\rangle=\int_{H_{x}}\langle y, x\rangle \lambda(d y) \\
= & \int_{S^{n-1}} \max \left(0,\left\langle y, g x_{0}\right\rangle\right) \lambda(d y) \\
& \quad\left(\text { where } g \in G \text { is such that } g x_{0}=x\right) \\
= & \int_{S^{n-1}} \max \left(0,\left\langle g^{-1} y, x_{0}\right\rangle\right) \lambda(d y)=\int_{S^{n-1}} \psi\left(g^{-1} y\right) \lambda(d y) \\
= & \int_{G} \psi\left(g^{-1} g^{\prime} x_{0}\right) \mu\left(d g^{\prime}\right)=\int_{\sigma^{\prime}} \psi\left(\left(g^{\prime}\right)^{-1} g x_{0}\right) \mu\left(d g^{\prime}\right) \\
= & \lambda^{*} \psi\left(g x_{0}\right)=\lambda^{*} \psi(x)
\end{aligned}
$$

where $\lambda^{*} \psi$ is the convolution product of $\lambda$ and $\psi$ on the symmetric space $S^{n-1}$. Thus the hypotheses of Lemma 2 guarantee that $\lambda^{*} \psi$ is a constant function on $S^{n-1}$ and so its Fourier coefficients vanish except at the spherical function $f_{0}^{n-1}$. Thus $0=\hat{\psi}\left(f_{k}^{n-1}\right) \hat{\lambda}\left(f_{k}^{n-1}\right)$ for $k$ 
a positive even integer, so on account of Lemma $6, \hat{\lambda}\left(f_{k}^{n-1}\right)=0$ for $k$ an even positive integer. This completes the proof of Lemma 2 .

\section{REFERENCES}

1. P. R. Halmos, The range of a vector measure, Bull. Amer. Math. Soc. 54 (1948), 416-421.

2. S. Helgason, Differential Geometry and Symmetric Spaces, New York, 1962.

3. R. P. Kaufman and N. W. Rickert, An inequality concerning measures, Bull. Amer. Math. Soc. 72 (1966), 672-676.

4. C. Müller, Spherical Harmonics, Berlin, 1966.

5. G. Schwarz, Variations on vector measures, Pacific J. Math. 23 (1967), 373-375.

Received August 22, 1966, and in revised form May 3, 1967. This research supported by Army Research Office (Durham).

YALE UNIVERSITY 



\section{PACIFIC JOURNAL OF MATHEMATICS}

\section{EDITORS}

\section{H. ROYDEN \\ Stanford University \\ Stanford, California}

\author{
J. P. JANS \\ University of Washington \\ Seattle, Washington 98105
}

J. DugundJI

Department of Mathematics

Rice University

Houston, Texas 77001

RichaRd ARENS

University of California

Los Angeles, California 90024

\section{ASSOCIATE EDITORS}
E. F. BECKENBACH
B. H. NeumanN
F. WOLF
K. YOSIDA

\section{SUPPORTING INSTITUTIONS}

\author{
UNIVERSITY OF BRITISH COLUMBIA \\ CALIFORNIA INSTITUTE OF TECHNOLOGY \\ UNIVERSITY OF CALIFORNIA \\ MONTANA STATE UNIVERSITY \\ UNIVERSITY OF NEVADA \\ NEW MEXICO STATE UNIVERSITY \\ OREGON STATE UNIVERSITY \\ UNIVERSITY OF OREGON \\ OSAKA UNIVERSITY \\ UNIVERSITY OF SOUTHERN CALIFORNIA
}

\author{
STANFORD UNIVERSITY \\ UNIVERSITY OF TOKYO \\ UNIVERSITY OF UTAH \\ WASHINGTON STATE UNIVERSITY \\ UNIVERSITY OF WASHINGTON

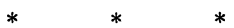 \\ AMERICAN MATHEMATICAL SOCIETY \\ CHEVRON RESEARCH CORPORATION \\ TRW SYSTEMS \\ NAVAL ORDNANCE TEST STATION
}

Mathematical papers intended for publication in the Pacific Journal of Mathematics should be typewritten (double spaced). The first paragraph or two must be capable of being used separately as a synopsis of the entire paper. It should not contain references to the bibliography. Manuscripts may be sent to any one of the four editors. All other communications to the editors should be addressed to the managing editor, Richard Arens at the University of California, Los Angeles, California 90024.

50 reprints per author of each article are furnished free of charge; additional copies may be obtained at cost in multiples of 50 .

The Pacific Journal of Mathematics is published monthly. Effective with Volume 16 the price per volume (3 numbers) is $\$ 8.00$; single issues, $\$ 3.00$. Special price for current issues to individual faculty members of supporting institutions and to individual members of the American Mathematical Society: $\$ 4.00$ per volume; single issues $\$ 1.50$. Back numbers are available.

Subscriptions, orders for back numbers, and changes of address should be sent to Pacific Journal of Mathematics, 103 Highland Boulevard, Berkeley 8, California.

Printed at Kokusai Bunken Insatsusha (International Academic Printing Co., Ltd.), 7-17, Fujimi 2-chome, Chiyoda-ku, Tokyo, Japan.

PUBLISHED BY PACIFIC JOURNAL OF MATHEMATICS, A NON-PROFIT CORPORATION

The Supporting Institutions listed above contribute to the cost of publication of this Journal, but they are not owners or publishers and have no responsibility for its content or policies. 


\section{Pacific Journal of Mathematics}

\section{Vol. 23, No. 2 \\ April, 1967}

Herbert Stanley Bear, Jr. and Bertram John Walsh, Integral kernel for

one-part function spaces .......................... 209

Mario Borelli, Some results on ampleness and divisorial schemes ....... 217

John A. Erdos, Unitary invariants for nests . ................... 229

Nathaniel Grossman, The volume of a totally-geodesic hypersurface in a pinched manifold.................................. 257

D. M. Hyman, A generalization of the Borsuk-Whitehead-Hanner theorem ............................................. 263

I. Martin (Irving) Isaacs, Finite groups with small character degrees and large prime divisors ............................. 273

I. Martin (Irving) Isaacs, Two solvability theorems ................ 281

William Lee Johnson, The characteristic function of a harmonic function in a locally Euclidean space ............................... 291

Ralph David Kopperman, Application of infinitary languages to metric spaces ............................................. 299

John Lauchlin MacDonald, Relative functor representability ............ 311

Mahendra Ganpatrao Nadkarni, A class of measures on the Bohr group.... 321

Keith Lowell Phillips, Hilbert transforms for the p-adic and p-series fields....................................... 329

Norman R. Reilly and Herman Edward Scheiblich, Congruences on regular semigroups ...

Neil William Rickert, Measures whose range is a ball ... . .

Gideon Schwarz, Variations on vector measures

Ronald Cameron Riddell, Spectral concentration for self-adjoint operators. .

Haskell Paul Rosenthal, A characterization of restrictions of

Fourier-Stieltjes transforms ................... 